\title{
Case-based User Profiling in a Personal Travel Assistant
}

\author{
Pawel Waszkiewicz, Pádraig Cunningham \\ Department of Computer Science \\ Trinity College Dublin \\ P.Waszkiewicz@cs.bham.ac.uk \\ Padraig.Cunningham@cs.tcd.ie
}

\author{
Ciara Byrne \\ Broadcom Éireann Research Ltd. \\ Dublin, Ireland. \\ cbe@broadcom.ie
}

\section{Introduction}

In this paper we present an architecture for a Personal Travel Assistant (PTA). We focus on the ability of this PTA to elaborate a user's travel requirements and evaluate offers. These decisions are based on a User Profile that has the interesting characteristic that it adopts a lazy policy on learning from a user's past behavior.

Information on user behaviour is stored in a case base in the form of a Case Retrieval Net (Lenz, Auriol \& Manago, 1998). A Retrieval Net can capture context-sensitive similarity information which is important in this domain. This lazy approach also has the advantages that it is incremental, extendible and allows flexible reuse of the information. In contrast, eager approaches must make assumptions about how the learned knowledge is to be used.

This user profiling system is being implemented as part of the Personal Travel Assistance scenario proposed by FIPA (Foundation for Intelligent Physical Agents).

\section{The FIPA PTA Scenario}

FIPA is a standards organisation concerned with specifying generic agent technologies. FIPA has proposed a set of prototype applications in which proposed standards can be tested. One of these applications is a travel scenario where agents assist a user both during pre-trip planning and during travel.

In the travel scenario envisaged, there will be three different types of agent: Personal Travel Agents (PTA) which represent the interests of an individual user or organisation, Travel Broker Agents (TBA) which broker travel services, and Travel Service Agents (TSA) which represent a particular travel service or have expertise in a particular area, e.g. Danish hotels. A user requests a trip by giving the PTA the trip details and specifying some preferences, such as preferred airline. The PTA then negotiates with the TBA over the details. The TBA composes a trip from several different elements (e.g. flight, hotel, etc.) supplied by the TSAs.

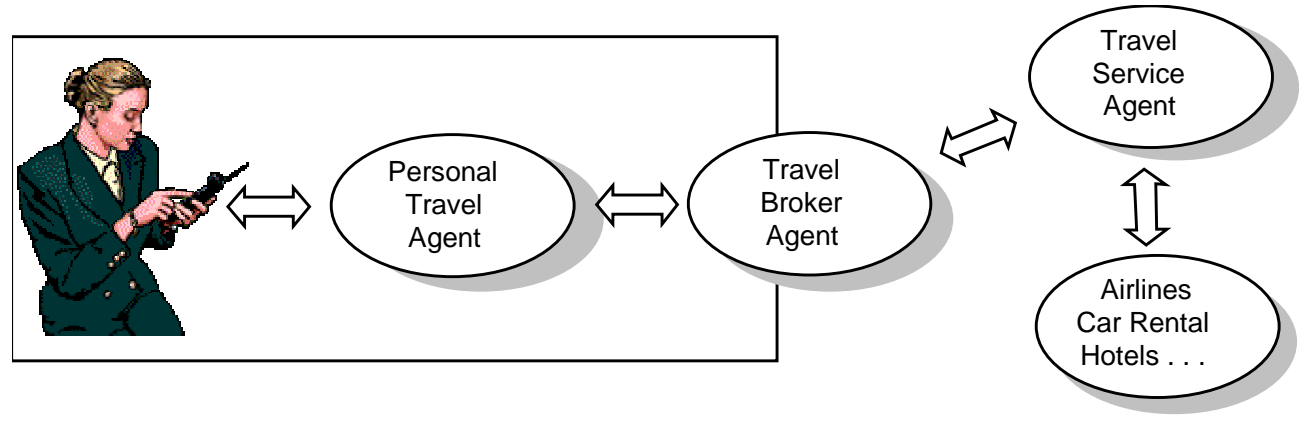

Figure 1. The Agent Interactions in the FIPA PTA scenario. 
This paper focuses on the design of the PTA and in particular on the pre-trip planning phase. We have identified two tasks which the PTA can learn to perform for the user during this phase: Adding extra user preferences to a travel request (Task 1) and evaluating an offer (Task 2).

\section{Lazy Learning}

Eager Learning is the dominant approach in Machine Learning. Decision Trees, Rule Induction, and Neural Networks are all eager in the sense that a hypothesis is cached during training and the data is discarded. Lazy learning techniques defer processing of their inputs until they receive requests for information, reply to information requests by combining their stored data and discard the constructed solution. Lazy learning systems often use local approaches (problems are solved by reference to nearby examples in the problem space) which can lead to highly adaptive behaviour not usually found in eager algorithms (Aha, 1997).

The PTA user profiling system can be considered as a partially lazy learner since it does not always discard a solution but may save it as a new case.

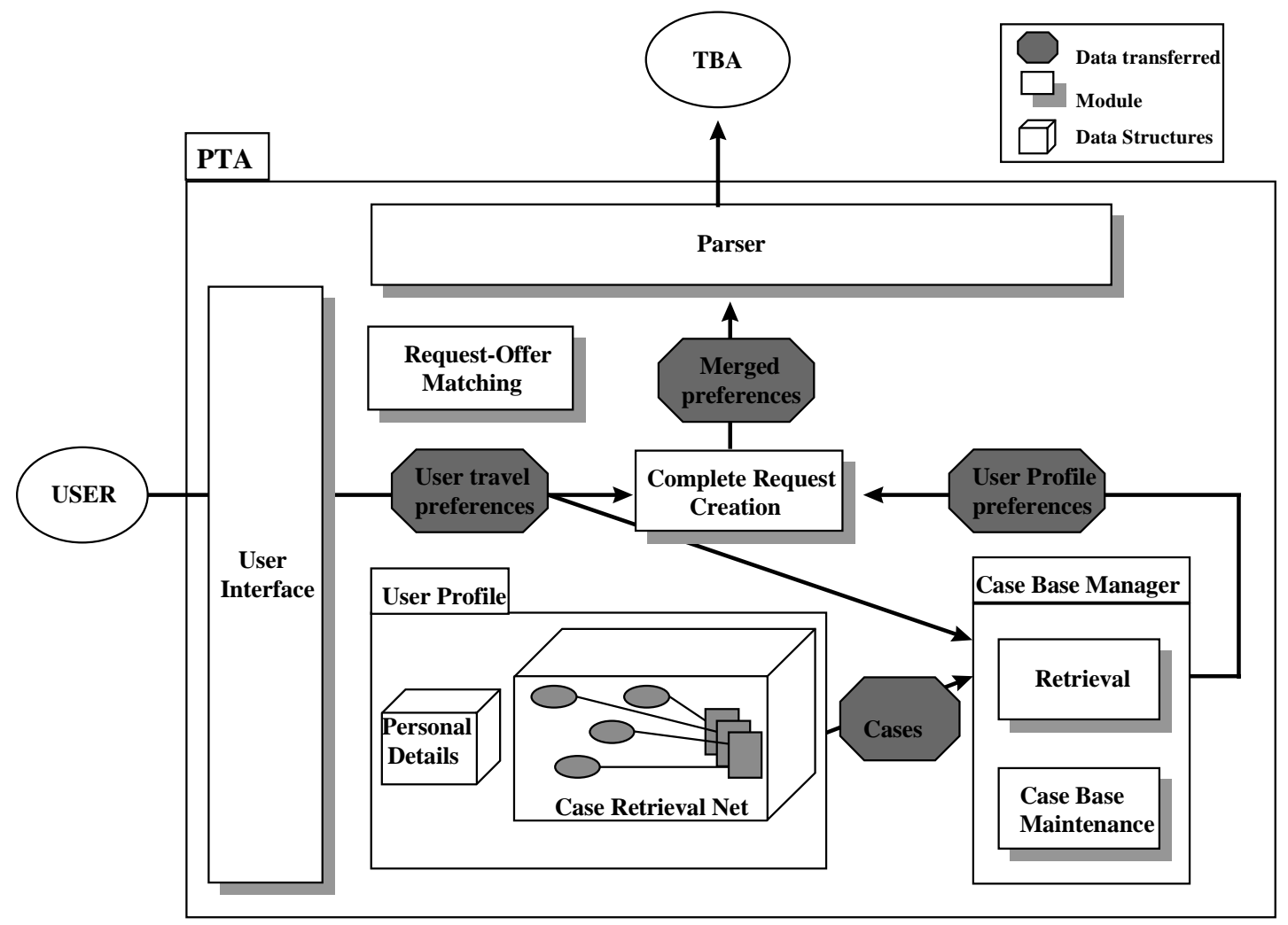

Figure 2. PTA Architecture

\section{Case-Based Reasoning within the PTA}

The PTA stores a user profile as a collection of cases. CBR was chosen as the learning method because it is an incremental learning system, cases can be acquired easily, the form of the profile learnt is flexible enough to be used in several different tasks and the decisions of 
the agent can be easily explained to the user.

The PTA acquires profile information by observing the user's actions when planning a trip. This information is converted into cases which are held in a Case Retrieval Net. A minimal amount of direct user feedback will also be used.

Figure 2 shows the flow of data through the system when the user requests a trip. The travel preferences supplied by the user are used to query the case base, and one or more cases similar to the new case are retrieved. Travel preferences defined in these cases are extracted. Preferences may be selected from several different cases making this an example of compositional adaptation. The travel preferences specified by the user and those extracted from the case base are merged. The PTA then constructs a trip request message from this information and sends it to the TBA. A new case is created based on this planning episode.

Task 2 involves evaluating an offer sent by the TBA whose features do not exactly match the preferences specified by the user. Some basic details in the offer and the request sent by the PTA, e.g. destinations, are compared to make sure that they match. If there is a mismatch between these details, then the offer is rejected immediately. Otherwise, the rest of the user's preferences are compared with the offer details and the differences between them are identified. This information is passed to the retrieval module which retrieves cases in which there were similar discrepancies. If the offer is categorised as being of interest (depending on whether the offer was accepted in the retrieved cases), then it is passed to the user for evaluation. Otherwise it is rejected.

\section{Conclusions \& Future Work}

We feel that this case-based approach to user profiling is suitable in this domain. The use of the Case-Retrieval Net allows the specification of context-sensitive similarity information and variability in the relevance of features to individual cases.

This system is currently under implementation in Java using ACL the Agent communication Language from FIPA and ASL an Agent Service Layer from Broadcom Éireann Research. (Kerr et al., 1998)

\section{References}

Aha, D. W. (1997). Editorial on Lazy Learning. Artificial Intelligence Review, 11, 7-10.

Lenz M., Auriol, E. and Manago, M. (1998) Diagnosis and Decision Support. In Lenz, M., Bartsch-Sporl, Burkhard H. D. and Wess S. (eds.) Case-Based Reasonng Technology. From Foundations to Applications, Springer (Lecture Notes in Computer Science; Vol. 1400: Lecture Notes in Artificial Intelligence)

FIPA (1997) Foundation for Intelligent Physical Agents, Draft Specification, Part 4, Personal Travel Assistance, 1997, http://www.fipa.org/

Kerr, D., O'Sullivan, D., Evans, R., Richardson, R., Somers, F., (1998) Experiences using Intelligent Agent Technologies as a Unifying Approach to Network and Service Management 5th International Conference on Intelligence in Services and Networks, May 25-28 1998, Antwerp, Belgium. 